\title{
237
}

\section{A class of enumerations of importance in genetics}

\author{
By R. A. Fisher, F.R.S.
}

(Received 21 June 1949)

\begin{abstract}
A number of enumerations arising in geneties, e.g. of sots of isomorphic genotypes, of modes of gamote formation, of the modes of formation of ordered and unordered tetrads, etc., have been found in previous work to yield solutions expressible as the mean powers of arbitrary degree of appropriate series of bases. These are such as to yield integral values of the mean for all integral exponents.

The general combinatorial theorem governing this class of enumeration is given in $\$ 2$. Other sections illustrate the method in operation by confirming and extending the genetic formulae so far established.

In $\$ 3 \cdot l$ is a brief discussion of a partitional function which serves to enumerate the numbers of partitions of any partible number in a lattice of arbitrary cimensionality.
\end{abstract}

\section{Illustrative examples}

During recent years a number of the problems encountered in genetic analysis have been found to involve problems of enumeration, the solutions of which were far from obvious, although structurally similar.

Among the first of these to have the solution published (Fisher 1947) were (i) the number of modes of gamete formation in tetrasomic organisms with $l$ linked loci, for which the formula

$$
\begin{aligned}
& \frac{1}{48} \cdot 16^{l}+\frac{1}{3} \cdot 4^{l}+\frac{1}{3}, \\
& 2, \quad 11, \quad 107, \quad 1451, \quad \ldots,
\end{aligned}
$$

was demonstrated by a somewhat indirect route. In the same paper the formula (ii)

$$
\begin{gathered}
\frac{1}{6 ! 3 !}\left(210^{l}+15.60^{l}+3.30^{l}+40.24^{l}+45.20^{l}+135.10^{l}+335.6^{l}+320.3^{l}+630.2^{l}\right), \\
2, \quad 40, \quad 3175, \quad \ldots,
\end{gathered}
$$

was offered tentatively for hexasomic forms. This has since been verified by the general method to be here discussed. In an appendix also was given without proof the enumeration (iii)

$$
\begin{gathered}
\frac{1}{24}\left(14^{l}+9.6^{l}+14.2^{l}\right), \\
4, \quad 24, \quad 200, \quad 2096, \quad \ldots,
\end{gathered}
$$

for the number of sets of isomorphic genotypes of a tetrasomic organism heterogenic at $l$ loci. No attempt was made at that time to enumerate the numbers for a hexasomic organism, for which, when $l$ is unity, the number is 10 , and when $l=2$, the number of sets was found to be 277. It will later be shown that the number using $l$ loci is in general

$$
\begin{gathered}
\frac{1}{6 !}\left(202^{l}+15.66^{l}+60.30^{l}+40.19^{l}+120.9^{l}+180.8^{l}+40.7^{l}+120.3^{l}+144 \cdot 2^{l}\right) \\
10, \quad 277,20343, \ldots,
\end{gathered}
$$


Apart from their genetical meaning, the two examples last given are in general combinatorial theory to be recognized as the numbers of partitions of the partible numbers 4 and 6 in $l$ dimensions.

Since the events of a single reduction division are capable of determining not a single gamete only, but a tetrad of two pairs of gametes, the joint content of which specifies more completely than a single gamete the course of the preceding events, it is relevant also to inquire as to the number of modes of tetrad formation. For disomic organisms this is shown without great difficulty to be

$$
\begin{gathered}
\frac{1}{16}\left(6^{l}+3.4^{l}+7.2^{l}\right), \\
2, \quad 7, \quad 29, \quad 136, \quad 692, \quad 3712, \quad \ldots .
\end{gathered}
$$

With tetrasomic or hexasomic organisms the problem is much more complex. The same is true of the specification of matings; e.g. those involving two disomic genotypes, supposing them to be not equivalent homozygotes at any of the loci concerned, or, in other words, ignoring all loci at which the mating is homogenic; then the number of mating types heterogenic at exactly $l$ loci is found to be

$$
\begin{aligned}
& \frac{1}{8}\left(14^{l}+5.6^{l}+2.2^{l}\right), \\
& 6, \quad 48, \quad 480, \quad 5616, \quad \ldots,
\end{aligned}
$$

The question might be asked equally of polysomic organisms, or in respect of two or more matings.

\section{Combinatorial structure common to this Class of PROblem}

Let us suppose that a formula is to be constructed by inserting appropriate 'contents' into a finite number of cells or ' containers'. Inherent in the data of each problem is a certain finite group of permutations among the containers, or among the elements of the contents. If any formula can be derived from another by a permutation of this basic group, the two formulae are said to belong to the same set; what is to be enumerated is the number of such sets.

It will be noticed that the formulae derived from any given formula by permutations of the basic group, by which the sets are defined, may be all different, in which case the set will contain a number of formulae equal to the order $N$ of the basic group, or alternatively that a subgroup of this group leaves the formula unaltered, and that in this case the number of different formulae in the set is reduced by a factor which is the order of the invariant subgroup.

If, starting with one member of each set, we apply all permutations of the group, including the identity, we shall develop an assemblage of formulae numbering in all $N$ times as many as the number of sets. In this assemblage every possible formula must occur, and the frequency of its occurrence is equal to the order of the subgroup by which it is unaltered.

Now the sum for all formulae of the orders of the subgroups of permutations by which they are unaltered is equal to the sum for all permutations of the numbers of formulae unaltered by them. Suppose, now, for any given permutation there are $a$ ways of inserting contents which shall be unaltered by this permutation, then, if the 
contents are added in $l$ successive layers, there must be $a^{l}$ invariant compound formulae possible. The number of sets is then given by

$$
\frac{1}{N} S\left(a^{l}\right)
$$

where $S$ stands for summation over all the $N$ permutations, including the identity, of the given group.

\section{OPERATIONAL PROCEDURE; ISOMORPHIC SETS OF GENOTYPES}

Consider as a first illustration the number of isomorphic sets of tetrasomic genotypes.

There are-four chromosomes, and the complete permutation group of these four generates members of the same set. The twenty-four elements of this group may be classified according to the partitions of 4 , as follows:

\begin{tabular}{rll}
1 & without change, the identity & $\left(1^{4}\right)$ \\
6 & interchanges of pairs & $\left(21^{2}\right)$ \\
3 & double interchanges of pairs & $\left(2^{2}\right)$ \\
8 & cycles of three & $(31)$ \\
6 & cycles of four & $(4)$ \\
\hline 24 & total &
\end{tabular}

The same partitions also can be used to specify the cell contents at any one locus; i.e. $\left(1^{4}\right)$ stands for the case of four different allelomorphic genes, $\left(21^{2}\right)$ for the case where two only are alike, $\left(2^{2}\right)$ for the case where there are two pairs of like genes, and (31) for the case of three alike and one different. The last case (4), representing four genes all alike, will be omitted, since the enumeration is to be in terms of the number of heterogenic loci, so that no homogenic locus need be considered.

The number of formulae of these kinds, equal to the number invariant with the identity, and the number invariant with the other classes of possible permutations, are set out in table 1.

TABLE I. NUMBer OF DISTRIbUtion FORMUlaE OF DIFFERENT PARTITIONAL TYPES INVARIANT FOR DIFFERENT TYPES OF PARTITION

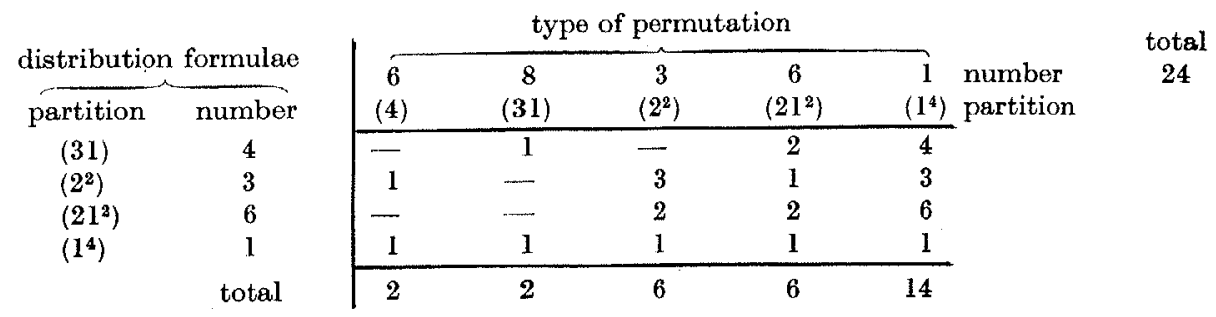

The number of distribution formulae for a given partition

is

$$
\begin{gathered}
P=\left(p_{1}^{\pi_{1}} p_{\mathbf{2}}{ }^{\pi_{2}} \ldots\right), \quad(p \pi)=n, \\
\frac{n !}{\left(p_{1} !\right)^{\pi_{1}} \ldots\left(\pi_{1} !\right) \ldots},
\end{gathered}
$$


appropriately to the fact that the interchange of any two equally numerous genes is recognized to be a matter of indifference; the numbers of permutations of different partitional types are, however,

$$
\frac{n !}{p_{1}^{\pi_{1}} \ldots\left(\pi_{1} !\right) \ldots}
$$

these add to $n$ ! Each is a permutation in $\pi_{1}$ cycles of $p_{1}, \pi_{2}$ cycles of $p_{2}$, etc.

The individual entries in the body of table 1 show for each of the five types of permutation for how many distribution formulae (of four different types in the four rows) each is inoperative. The sums of the five columns show that

For 1 permutation (the identity) there are 14 invariant formulae,

For 9 permutations there are 6 invariant formulae,

For 14 permutations there are 2 invariant formulae.

Since the permutation group of four objects is of order 24 , the enumeration formula is now obtained as

$$
\frac{1}{24}\left(14^{l}+9 \cdot 6^{l}+14 \cdot 2^{l}\right)
$$

giving the number of partitions in (exactly) $l$ dimensions of the partible number 4, or, the number of sets of isomorphic genotypes in a tetrasomic organism heterogenic at (exactly) $l$ loci.

For partitions of 6 , or for hexasomic genotypes, we have, instead of table 1, a similar table of ten rows and eleven columns, shown below as table 2 . giving, as previously stated, the enumeration

$$
\begin{gathered}
\frac{1}{720}\left(202^{l}+15.66^{l}+60.30^{l}+40.19^{l}+120.9^{l}+180.8^{l}+40.7^{l}+120.3^{l}+144 \cdot 2^{l}\right) \\
10, \quad 277, \quad 20343, \quad \ldots,
\end{gathered}
$$

In constructing the table it is a convenient check that the products of the entries in each row with the numbers at the head of the columns add in each case to 720 .

The table for partitions of 8 is shown in table 3 . The work is heavier here, though most of the entries can be written down at sight. The enumeration formula is, however, not very lengthy, as it has only 19 terms. It may be written in the form

$$
\begin{gathered}
\frac{1}{40320} \cdot 4139^{l}+\frac{1}{1440} \cdot 1079^{l}+\frac{1}{192} \cdot 351^{l}+\frac{1}{360} \cdot 254^{l}+\frac{5}{384} \cdot 163^{l}+\frac{1}{36} \cdot 86^{l} \\
+\frac{1}{96} \cdot 81^{l}+\frac{1}{24} \cdot 42^{l}+\frac{3}{32} \cdot 37^{l}+\frac{1}{36} \cdot 32^{l}+\frac{1}{36} \cdot 20^{l}+\frac{1}{30} \cdot 19^{l}+\frac{1}{32} \cdot 15^{l} \\
+\frac{1}{12} \cdot 12^{l}+\frac{1}{8} \cdot 10^{l}+\frac{1}{10} \cdot 9^{l}+\frac{1}{15} \cdot 4^{l}+\frac{1}{8} \cdot 3^{l}+\frac{1}{7} \cdot 2^{l}
\end{gathered}
$$

The numerical values are

$$
21, \quad 2974, \quad 2991002, \quad \ldots
$$

\subsection{Properties of a special partitional function}

In connexion with this mode of enumerating the number of partitions of any partible number in $l$ dimensions, it may be noted that the general result may be expressed in terms of two partitional functions.

If $P$ stands for any partition of the partible number $n$

$$
P=\left(p_{1}^{\pi_{1}} p_{2}^{\pi}, \ldots\right), \quad \Sigma(p \pi)=n,
$$




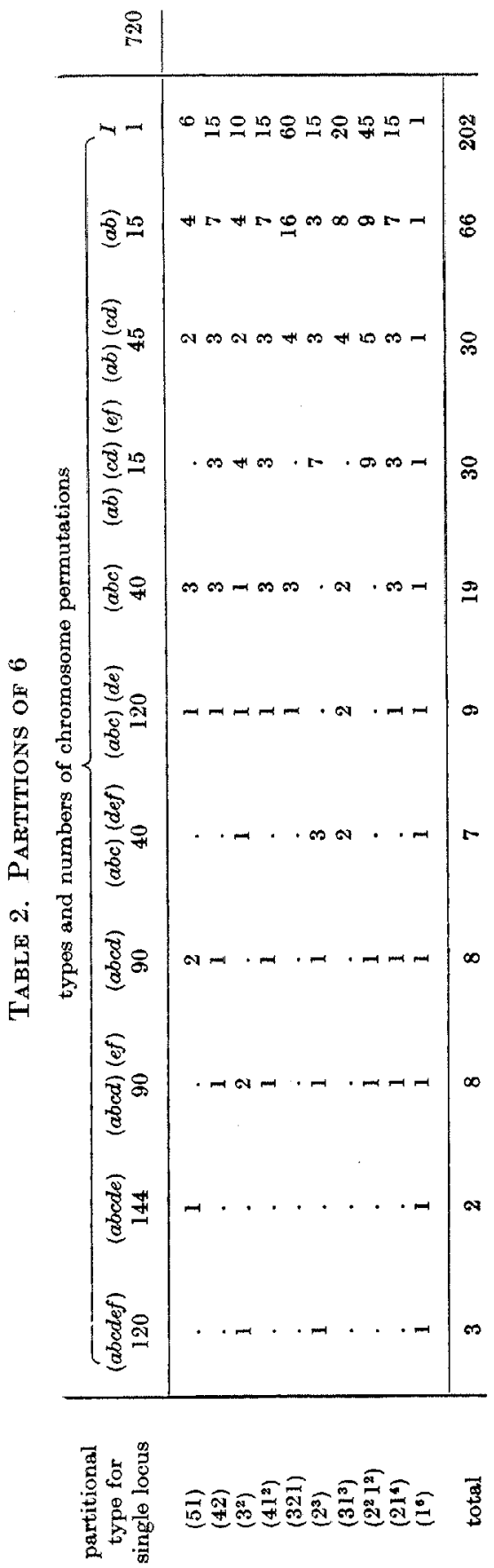




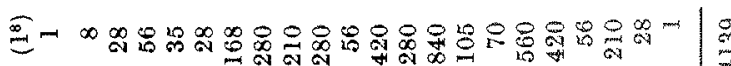

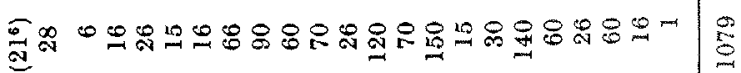

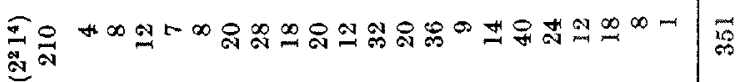

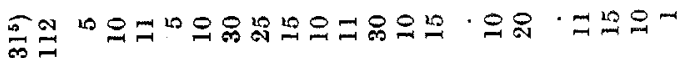

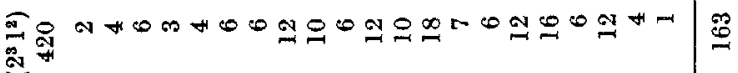

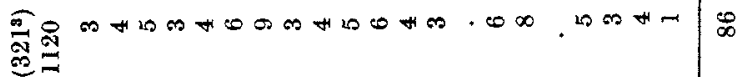
登条

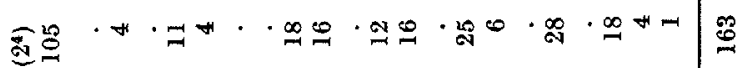

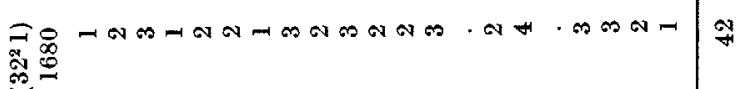

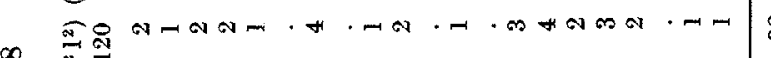



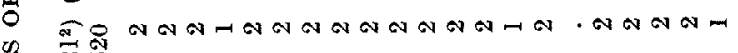
密

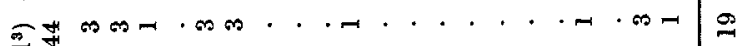

[1

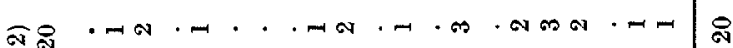
ฝै

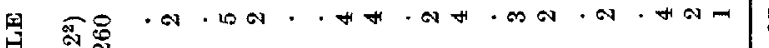

状学

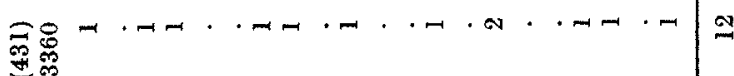

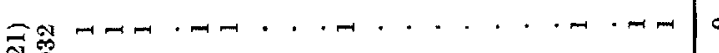

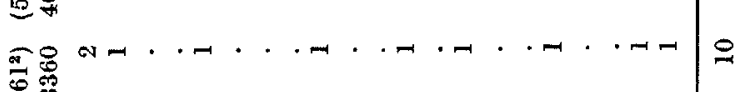

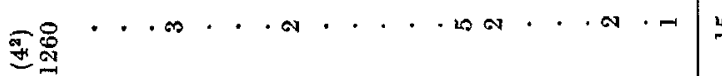

$\mathbb{B}_{0}^{\infty} \infty$

สํํ유.

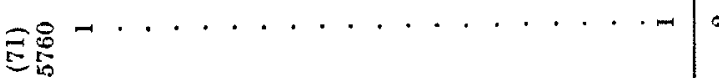
œ

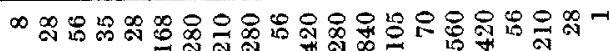

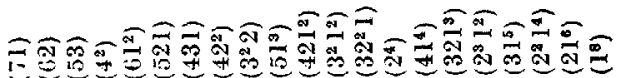


and $\alpha(P)$ stands for the partitional function

$$
\alpha(P)=\frac{n !}{p_{1}^{\pi_{1}} \ldots \pi_{1} ! \ldots},
$$

then $\alpha(P)$ enumerates the numbers of permutations of given partitional type.

The method of $\S 3$ has also defined a second partitional function, which we may denote by $e(P)$, such that the number of partitions of $n$ in $l$ dimensions is

$$
\sum_{P / n}(e-1)^{l} \alpha / \sum_{P / n}(\alpha)
$$

in which the summation is taken over all partitions in 0 or 1 dimension of the number $n$, so that $\Sigma(\alpha)=n !$. The number of partitions in 0 or I dimension is therefore

$$
1+\frac{\Sigma(e-1) \alpha}{\Sigma(\alpha)}=\Sigma(e \alpha) / \Sigma(\alpha)
$$

or the average value of $e$ over all permutations.

For small partitions the numerical value of $e$ may be obtained more expeditiously than by the construction of the bipartitional table, for when $P=\left(1^{n}\right)$ it is seen that $e$ is the coefficient of $x^{n} / n !$ in the expansion in powers of $x$ of

The series of coefficients is

$$
F(x)=\mathrm{e}^{\mathrm{e}^{x}-1}
$$

$$
1, \quad 1,2, \quad 5, \quad 15,52,203,877,4140,
$$

up to the eighth degree.

Given the contents of any partition we may build up a differential operator competent to give the partitional function $e$, by acting upon $F(x)$. For unit parts we need only insert a factor $D$ for each part; for prime parts a factor $(D+1)$; for compound parts having $f$ factors (excluding unity), the factor is $(D+f)$. If, then, no two parts have a common factor, the differential operator is complete. Thus for the partition $\left(321^{3}\right)$ we have the operator

$$
D^{3}(D+1)^{2}
$$

yielding

$$
52+2(15)+5=87,
$$

in accordance with the entry [giving $(e-1)$ ] in table 3 .

When some of the parts have common factors we have to introduce also the sums of the factors common to any two parts, the sums of the squares of the factors common to any three, and so on. So for the partition $P=\left(6^{2}\right)$, we find

$$
(D+3)^{2}+11=D^{2}+6 D+20,
$$

and for $P=\left(2^{3}\right)$ the operator is

$$
(D+1)^{3}+3.2 \cdot(D+1)+4=D^{3}+3 D^{2}+9 D+11,
$$

giving

$$
e(P)=5+6+9+11=31,
$$

in accordance with the entry in table 2 .

* The operational procedure as stated here is not entirely correct. See J.H. Bennett (1956) Partitions in more than one dimension. Journal of the Royal Statistical 


\section{Modes of gamete formation}

Consider a tetrasomic organism having (of any particular kind) four homologous chromosomes, $A, B, C$ and $D$. Thë gametes will be diploid, and will contain for each locus two representative genes. A gamete may then be represented by

\section{$A A \quad 4$ formulae, \\ $A B \quad 12$ formulae.}

Gametes of the first kind are said to exhibit double reduction at the locus in question. However many loci are considered, the gamete represented by any formula will be said to have originated by the same mode of gamete formation as that represented by any formula derivable from the first:

(a) by reversal of the symbols for the gametic chromosomes, an operation which may be represented by the symbol $r$,

(b) by permutation of the parental chromosomes, represented by such symbols as $(a b),(a b c),(a b)(c d)$, etc., 24 in all, including the identity $I$.

It is now easy to consider for each of the 48 possible operations, how many of the 16 formulae specified above are invariant.

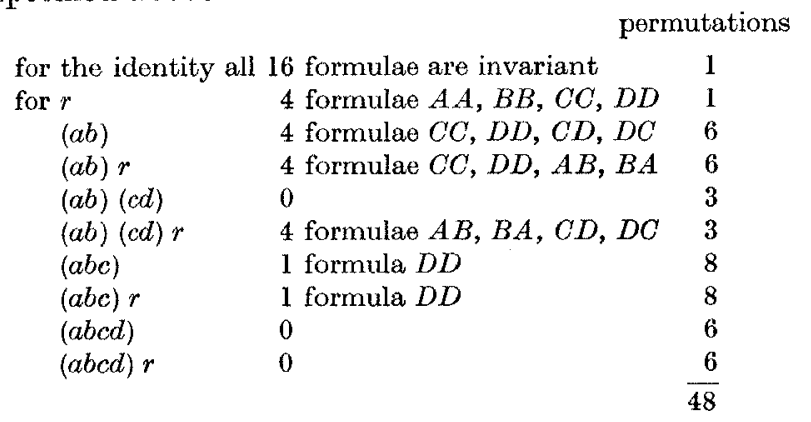

The formula enumerating the modes of gamete formation is therefore

or, more simply,

$$
\frac{1}{48}\left(16^{l}+16.4^{l}+16\right),
$$

where

$$
\begin{gathered}
3 a_{l}^{2}-1, \\
a_{l}=\frac{1}{12}\left(4^{l}+8\right) \\
a_{l}=4 a_{l-1}-2
\end{gathered}
$$

is easily calculated, since

$$
\begin{array}{rrrrrrrr}
l & 1 & 2 & 3 & 4 & 5 & 6 & \\
a & 1 & 2 & 6 & 22 & 86 & 342 & \\
3 a^{2}-1 & 2 & 11 & 107 & 1451 & 22187 & 350891 & \ldots
\end{array}
$$

By the methods previously employed the enumeration of the modes of gamete formation in hexasomic organisms presented a very intricate problem, and I had little confidence in the formula given. The method of this paper gives the same result by a much simpler path. The two modes of formation for a single locus are easily seen to give the two types of formula:

$\begin{array}{cc}\text { type } & \text { number of formulae } \\ A B C & 120 \\ A A B & 90\end{array}$


The number of these is to be ascertained for a group of permutations involving changes of two kinds. (i) Permutations of the position in which the three elements of the gamete are written down. These are six in number, being the identity $(I)$, three interchanges of pairs (12), (23), (31), and two cyclic changes (123), (132). (ii) There are the $6 !$ permutations of the six letters $a, b, c, d$, e and $f$, standing for the parental chromosomes. The whole group of $6 ! 3$ ! changes is exhibited in a two-way table, in which the number of invariant members of each class of formula have been entered separately.

Table 4. NUmbers of gametic formulae invariant for various PERMUTATIONS

$$
\begin{aligned}
& \text { permutations } \\
& \text { of letters } \\
& \text { (parental } \\
& \text { chromosomes) }
\end{aligned}
$$

\begin{tabular}{|c|c|c|c|}
\hline \multicolumn{3}{|c|}{$\begin{array}{l}\text { permutations of order (gametic } \\
\text { chromosomes) }\end{array}$} & \multirow[b]{2}{*}{$\begin{array}{c}\text { number } \\
\text { type }\end{array}$} \\
\hline$I$ & $\begin{array}{c}3 \\
(12)\end{array}$ & $\begin{array}{c}2 \\
(123)\end{array}$ & \\
\hline 120,90 & 0,30 & 0,0 & \\
\hline 24,36 & 8,12 & 0,0 & \\
\hline 0,6 & 8,2 & 0,0 & \\
\hline 6,18 & 0,6 & 3,0 & \\
\hline 0,0 & 0,0 & 0,0 & \\
\hline 0,0 & 2,0 & 3,0 & \\
\hline 0,6 & 0,2 & 0,0 & \\
\hline 0,0 & 0,0 & 6,0 & \\
\hline 0,0 & 0,0 & 0,0 & \\
\hline 0,0 & 0,0 & 0,0 & \\
\hline 0,0 & 0,0 & 0,0 & \\
\hline
\end{tabular}

$\begin{array}{rl}1 & I \\ 15 & (a b) \\ 45 & (a b)(c d) \\ 40 & (a b c) \\ 15 & (a b)(c d)(e f) \\ 120 & (a b c)(d e) \\ 90 & (a b c d) \\ 40 & (a b c)(d e f) \\ 90 & (a b c d)(e f) \\ 144 & (a b c d e) \\ 120 & (a b c d e f)\end{array}$

Adding the numbers for the two types of formulae, it appears that

1 permutation is inoperative for 210 formulae

15 permutations are inoperative for 60 formulae

3 permutations are inoperative for 30 formulae

40 permutations are inoperative for 24 formulae

45 permutations are inoperative for 20 formulae

135 permutations are inoperative for 10 formulae

335 permutations are inoperative for 6 formulae

320 permutations are inoperative for 3 formulae

630 permutations are inoperative for 2 formula $\theta$

giving the formula stated above, equivalent to

$$
\begin{gathered}
\frac{1}{4320} 210^{l}+\frac{1}{288} 60^{l}+\frac{1}{1440} 30^{l}+\frac{1}{108} 24^{l}+\frac{1}{96} 20^{l}+\frac{1}{32} 10^{l}+\frac{67}{864} 6^{l}+\frac{2}{27} 3^{l}+\frac{7}{48} 2 l . \\
2, \quad 40, \quad 3175, \quad \ldots .
\end{gathered}
$$

Tetrasomic gametes from octosomic parents may be of three kinds

$$
\begin{aligned}
& A B C D \text { with } 1680 \text { formulae } \\
& A A B C \text { with } 2016 \text { formulae } \\
& A A B B \text { with } 168 \text { formulae }
\end{aligned}
$$

Table 5 shows in columns corresponding with permutations of the gametic chromosomes, and rows with permutations of the parental chromosomes, the number of formulae invariant to each type. Since no formula is unaltered by some of the permutations of the parental chromosomes, these have been omitted. 
Table 5. Numbers of gametro formula invariant for Various PERMUTATIONS SEGREGATING IN OCTOSOMICS

$\begin{array}{rl}1 & I \\ 28 & (a b) \\ 210 & (a b)(c d) \\ 112 & (a b c) \\ 420 & (a b)(c d)(e f) \\ 1120 & (a b c)(d e) \\ 420 & (a b c d) \\ 105 & (a b)(c d)(e f)(g h) \\ 1680 & (a b c)(d e)(f g) \\ 1120 & (a b c)(d e f) \\ 2520 & (a b c d)(e f) \\ 1344 & (a b c d e) \\ 1120 & (a b c)(d e f)(g h) \\ 1260 & (a b c d)(e f)(g h) \\ 3360 & (a b c d)(e f g) \\ 4032 & (a b c d e)(f g) \\ 3360 & (a b c d e f) \\ 1260 & (a b c d)(e f g h) \\ 3360 & (a b c d e f)(g h)\end{array}$

\begin{tabular}{|c|c|c|c|c|}
\hline $\begin{array}{l}1 \\
I\end{array}$ & $\begin{array}{c}6 \\
(12)\end{array}$ & $\begin{array}{c}3 \\
(12)(34)\end{array}$ & $\begin{array}{c}8 \\
(123)\end{array}$ & $\begin{array}{c}6 \\
(1234)\end{array}$ \\
\hline 3864 & 392 & 56 & & \\
\hline 1170 & 222 & 58 & . & 2 \\
\hline 204 & 100 & 60 & . & 4 \\
\hline 540 & 80 & 20 & 15 & . \\
\hline 6 & 26 & 62 & $\cdot$ & 6 \\
\hline 54 & 30 & 22 & 9 & 2 \\
\hline 204 & 36 & 12 & . & 4 \\
\hline 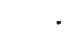 & & 64 & $\cdot$ & 8 \\
\hline . & 4 & 24 & 3 & 4 \\
\hline 6 & 2 & 2 & 12 & . \\
\hline 6 & 10 & 14 & r & 6 \\
\hline 54 & 12 & 6 & . & . \\
\hline$\bullet$ & $\cdot$ & 4 & . & 2 \\
\hline . & . & 16 & $\theta$ & 8 \\
\hline$\cdot$ & . & $\cdot$ & 3 & 4 \\
\hline 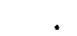 & 2 & 8 & . & 2 \\
\hline 6 & 2 & 2 & . & 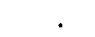 \\
\hline 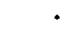 & $\cdot$ & $\cdot$ & . & 8 \\
\hline . & . & 4 & . & 2 \\
\hline
\end{tabular}

The enumerating function, in 31 terms, is then:

$\begin{array}{rrrr}3864^{l} & & & \\ 28.1170^{l} & 1260.62^{l} & 5040.24^{l} & 15120.10^{l} \\ 112.540^{l} & 630.60^{l} & 3360.22^{l} & 8960.9^{l} \\ 6.392^{l} & 84.58^{l} & 336.20^{l} & 27846.8^{l} \\ 168.222^{l} & 3.56^{l} & 3780.16^{l} & 29092.6^{l} \\ 630.204^{l} & 2464.54^{l} & 896.15^{l} & 57540.4^{l} \\ 1260.100^{l} & 2520.36^{l} & 7560.14^{l} & 40320.3^{l} \\ 672.80^{l} & 6720.30^{l} & 18284.12^{l} & 122472.2^{l} \\ 315.64^{l} & 2520.26^{l} & & \end{array}$

all divided by $8 ! 4$ !, or 967680 .

The numerical values, when $l$ is 1,2 and 3 , are 3,188 and 135188 .

\section{Tetrad FORMATION}

Another case in which the number of formulae is not so great that they cannot easily be examined individually is that of tetrad formation in diploid organisms. If all four products of the same meiosis can be observed, and if the pair of products of the first division can be distinguished, we have an ordered tetrad, with formulae of two kinds:

$$
\begin{array}{cc}
\text { type of formula } & \text { number } \\
A, A ; a, a & 2 \\
A, a ; A, a & 4
\end{array}
$$

The permutation group by which representations of the same mode of tetrad formation can be recognized consists of (i) two interchanges within the pairs of 
products of the second division, which may be represented by the symbols $p, q$; (ii) interchange between the products of the first division, represented by $r$; (iii) interchange of the gene symbols $(A a)$. The group is of order 16 .

Then it is easy to verify table 6 , showing the number of formulae invariant for any combination of these operations.

TABle 6. NUMBER OF FORMULAE FOR ORDERED TETRADS INVARIANT FOR DIFFERENT INTERCHANGES

\begin{tabular}{|c|c|c|c|}
\hline \multirow{2}{*}{\multicolumn{2}{|c|}{$\begin{array}{l}\text { reversals of } \\
\text { position }\end{array}$}} & \multicolumn{2}{|c|}{ gene interchange } \\
\hline & & $I$ & $\overrightarrow{(A a)}$ \\
\hline 1 & $I$ & 6 & - \\
\hline 2 & $p, q$ & 2 & 一 \\
\hline 1 & $p q$ & 2 & 4 \\
\hline 1 & $r$ & 2 & 4 \\
\hline 2 & $r p, r q$ & - & 2 \\
\hline 1 & $r p q$ & 2 & 4 \\
\hline
\end{tabular}

The number of modes of formation of ordered tetrads is therefore

$$
\begin{gathered}
\frac{1}{18}\left(6^{l}+3.4^{l}+7.2^{l}\right), \\
2, \quad 7, \quad 29, \quad 136, \quad 692, \quad \ldots .
\end{gathered}
$$

If the products of the second division are not recognizable, we shall have an unordered tetrad. Here there is only one type of formula

$$
\text { AAaa, }
$$

which can be written in 6 ways. Permutation among these four elements, in 24 ways, together with interchange of the gene symbols gives a group of order 48, with invariance shown by table 7 .

\begin{tabular}{|c|c|c|c|}
\hline \multirow{2}{*}{\multicolumn{2}{|c|}{$\begin{array}{l}\text { permutations } \\
\text { of position }\end{array}$}} & \multicolumn{2}{|c|}{ gene interchange } \\
\hline & & $I$ & $\widehat{(A a)}$ \\
\hline 1 & $I$ & 6 & - \\
\hline 6 & $(a b)$ & 2 & - \\
\hline 3 & $(a b)(c d)$ & 2 & 4 \\
\hline 6 & $(a b c d)$ & - & 2 \\
\hline
\end{tabular}

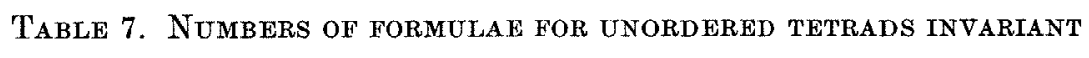
FOR.DIFFERENT PERMUTATIONS

The number of modes of formation for unordered tetrads is therefore

$$
\begin{gathered}
\frac{1}{48}\left(6^{l}+3.4^{l}+15.2^{l}\right), \\
1, \quad 3, \quad 11, \quad 48, \quad 236, \quad \ldots
\end{gathered}
$$

With ordered tetrads for tetrasomic organisms, the basic group contains four additional interchanges between the chromosomes of the four diploid gametes, while the gene interchange is replaced by a group of 24 permutations of the parental chromosomes. The order of the group is thus $2^{7} .4 !$. 
* I find this case very confusing, and have not thoroughly checked the result. It is put on record as an aid to any later worker who may consider the problem:

$$
\begin{aligned}
& \frac{4^{l}}{2^{7} .4 !}\left(630^{l}+4.90^{l}+51.24^{l}+10.18^{l}+102.12^{l}+161.6^{l}+252.4^{l}+24.3^{l}+84.2^{l}+3.12\right) \\
& 8,2538, \ldots .
\end{aligned}
$$

The unordered tetrads will be about one-third as numerous.

\section{ReFerence}

Fisher, R. A. 1947 The theory of linkage in polysomic inheritance. Phil. Trans. B, 233, 55-87.

* See J.A.Sved (1963) Modes of tetrad formation in tetrasomic inheritance. Heredity, $18,433-449$. 